\title{
SHOCK-LAYER BOUNDS FOR A SINGULARLY PERTURBED EQUATION
}

\author{
BY \\ JEFFREY S. SCROGGS* \\ Department of Mathematics, North Carolina State University, Raleigh, North Carolina
}

\begin{abstract}
The size of the shock-layer governed by a conservation law is studied. The conservation law is a parabolic reaction-convection-diffusion equation with a small parameter multiplying the diffusion term and convex flux. Rigorous upper and lower bounding functions for the solution of the conservation law are established based on maximum-principle arguments. The bounding functions demonstrate that the size of the shock-layer is proportional to the parameter multiplying the diffusion term.
\end{abstract}

1. Introduction. Upper and lower bounding functions are presented. The bounds demonstrate that, after the shock-layer has formed, the layer in solutions to the singularly perturbed hyperbolic partial differential equation

$$
P[u]:=\frac{\partial u}{\partial t}+\frac{\partial F(u)}{\partial x}-\varepsilon \frac{\partial^{2} u}{\partial x^{2}}-R(u)=0
$$

has width of size $O(\varepsilon)$. The analysis is performed in the style of Howes [2, 3, 4]. It begins with a multiple-scales asymptotic analysis. This provides the appropriate local scalings and indicates candidate forms for a bounding function. A bounding function is constructed from these candidates. Maximum principle arguments are then used to rigorously establish bounds for the solution. In this way, upper and lower bounds for solutions to Eq. (1) are established for any (not necessarily small) $\varepsilon$.

This presentation will concentrate on a comparison between solutions to (1) and solutions to the corresponding reduced equation

$$
P_{0}[U]:=\frac{\partial U}{\partial t}+\frac{\partial F(U)}{\partial x}-R(U)=0
$$

obtained by setting $\varepsilon=0$. The result will be a bound on the difference between the solution to this reduced equation and the solution to equation (1).

There are implications of this analysis for the computational aspects of the problem as well as for the physics modeled by conservation laws. The bounding functions

Received August 6, 1992.

1991 Mathematics Subject Classification. Primary 35B35, 35B25.

* Research conducted at ICASE supported by NASA Contract No. NAS NAS1-18605.

(C)1995 Brown University 
result in an upper bound on the size of the shock-layer. They isolate the internal-layer region in which viscosity is important from the convection-dominated outer region. This validates the assumption that the lack of resolution of the physics in the shocklayer affects the solution in smooth regions very little under certain circumstances; thus, the hyperbolic Eq. (2) may be substituted for Eq. (1).

This work extends well-known results for Burgers' equation, and sharpens the bounds of Howes [3,5] which apply in a more general setting.

2. Problem specification. This paper is concerned with presenting bounds for the solution $u$ to the quasilinear parabolic Eq. (1) on the domain

$$
D:=\{(x, t) \mid 0 \leq x \leq b, 0 \leq t<T\},
$$

subject to

$$
\begin{gathered}
u(x, 0)=g(x), \quad 0<x<b \\
u(0, t)=\alpha(t), \quad 0<t<T
\end{gathered}
$$

and

$$
u(b, t)=\beta(t), \quad 0<t<T .
$$

Let the portion of the boundary along which the data is specified be denoted by

$$
\partial D:=\{(x, t) \mid 0 \leq x \leq b, t=0\} \cup\{(x, t) \mid 0 \leq t<T, x=0, b\} .
$$

The boundary and initial data are continuous and sufficiently smooth so that $u$ is uniquely defined [1]. In particular, the boundary data $\alpha$ and $\beta$ have derivatives bounded independently of $\varepsilon$. Corner-layers in $u$ are prohibited by assuming the compatibility conditions

$$
\begin{gathered}
\alpha(0)=g(0), \quad g(b)=\beta(0), \\
\frac{d \alpha}{d t}+\frac{d}{d x} F(g)-R(g)=0 \quad \text { at }(x, t)=(0,0),
\end{gathered}
$$

and

$$
\frac{d \beta}{d t}+\frac{d}{d x} F(g)-R(g)=0 \quad \text { at }(x, t)=(b, 0) .
$$

For simplicity, it is assumed that all boundaries are inflow boundaries; hence, $\alpha(t) F^{\prime}(\alpha(t))>0$ and $\beta(t) F^{\prime}(\beta(t))<0$. Also assume that there is a single shock-layer in the initial data that is contained in an $O(\varepsilon \ln (\varepsilon))$ neighborhood $\pi_{0}$ of $\left(\Gamma_{0}, 0\right)$, where $\Gamma_{0}$ is the location of the shock in $U$ at $t=0$. The domain of the initial viscous-layer is $\pi_{0}:=\left\{(x, t) \mid t=0\right.$ and $\left.\left|x-\Gamma_{0}\right|<\varepsilon \ln (\varepsilon)\right\}$.

The solutions to the parabolic problem will be compared to a weak solution of the hyperbolic Eq. (2). Let $U$ be the weak solution of (2) with boundary data (5-6), that is, the solution to (1) in the limit as $\varepsilon>0$ tends to zero (denoted as $\varepsilon \downarrow 0$ ). The initial condition will reflect a shock emanating from $(t, x)=\left(0, \Gamma_{0}\right)$. Thus, the initial condition for $U$ is

$$
U(x, 0)=g_{0}(x), \quad 0<x<b,
$$

where the difference $g(x)-g_{0}(x)$ is zero except when $\left|x-\Gamma_{0}\right|<\varepsilon \ln \varepsilon$. The relationship between $g$ and $g_{0}$ will be discussed in more detail in the proof of Theorem 4.4. 
Let the path of the shock in $U$ be given by the curve $(x, t)=(\Gamma(t), t)$. It is natural to describe the values of $U$ at the shock as

$$
U_{R}(t)=\lim _{x \downarrow \Gamma(t)} U(x, t)
$$

and

$$
U_{L}(t)=\lim _{x \uparrow \Gamma(t)} U(x, t) .
$$

For ease of presentation we will assume that $U_{L}>U_{R}$. The solution $U$ to (2) will satisfy the entropy condition

$$
f\left(U_{L}(t)\right)>S(t)>f\left(U_{R}(t)\right)
$$

where $f(U)=F^{\prime}(U)$, and the speed $S(t)$ of the shock is given by the RankineHugoniot jump condition [6]

$$
S(t)=\frac{F\left(U_{L}(t)\right)-F\left(U_{R}(t)\right)}{U_{L}(t)-U_{R}(t)} .
$$

The entropy condition may be written as

$$
\mu(t)=U_{L}(t)-U_{R}(t) \geq \mu_{0}
$$

for a constant $\mu_{0}>0$ that is independent of $\varepsilon$. It is assumed that the shock is part of the initial data and exists for the entire domain considered; thus, $\mu(t)$ is defined for all $t>0$.

3. Asymptotic analysis. The discussion includes introducing the appropriate scales and equations when Eq. (1) is used to model a shock-layer. These results are obtained using a heuristic analysis that treats $\varepsilon$ as a small parameter. The results are exploited in the construction of the bounding function and are made more rigorous in $\S 4$. First, some of the properties of the inviscid solution will be discussed.

3.1. Derivation of bounding function. In this section we will derive a canonical form for the bounding function. The assumptions utilized in the derivation are used only to motivate the equation governing the canonical form. The validity of the bounding function independent of these assumptions will be established when it is used in $\S 4$.

A canonical form for the solution of the viscous problem is derived here. Large gradients in the neighborhood of a shock-layer are resolved by using the spatial scale $1 / \varepsilon$. This scale is combined with shock-following to obtain the internal-layer coordinates

$$
\zeta=\frac{x-\Gamma}{\varepsilon} \text { and } \tau=t
$$

The transformation defined by these coordinates is applied to Eq. (1) to obtain

$$
\frac{\partial F(\hat{u})}{\partial \zeta}-S(t) \frac{\partial \hat{u}}{\partial \zeta}-\frac{\partial^{2} \hat{u}}{\partial \zeta^{2}}=\varepsilon\left(\frac{\partial \hat{u}}{\partial t}+R(\hat{u})\right),
$$

where $\hat{u}(\zeta, \tau)=u(x, t)$. This suggests the regular expansion

$$
\hat{u}=\hat{u}_{0}+\varepsilon \hat{u}_{1}+\cdots
$$


for $\hat{u}$. We assume that this expansion is a priori valid in the shock-layer and use identification in $\varepsilon$ to obtain the equation

$$
\frac{\partial F\left(\hat{u}_{0}\right)}{\partial \zeta}-S(t) \frac{\partial \hat{u}_{0}}{\partial \zeta}-\frac{\partial^{2} \hat{u}_{0}}{\partial \zeta^{2}}=0
$$

for $\hat{u}_{0}$. This equation is integrated with respect to $\zeta$ once to obtain

$$
F\left(\hat{u}_{0}\right)-S(t) \hat{u}_{0}-\frac{\partial \hat{u}_{0}}{\partial \zeta}=\text { constant. }
$$

To make this equation easier to solve, we approximate $S-F / \hat{u}_{0}$ by $\kappa \zeta^{P}$, and we set the constant in the right-hand side of the above equation to zero. The solution to this equation is the exponential

$$
\exp \left(-\kappa \zeta^{p+1}\right)
$$

Since the magnitude of the bounding function should be decaying away from the shock-layer, we assume $\kappa>0$, and substitute $|\zeta|$ for $\zeta$. This is the candidate form for the bounding function.

Guided by the form (14), let the general form for the bounding function $\omega$ be

$$
\omega=a \nu t+\theta(t) \exp \left(-K \hat{x}^{p}\right) .
$$

Here $\hat{x}(\Gamma(t), x)$ is a linear measure of the distance between $(x, t)$ and $(\Gamma(t), t)$. The power of the distance function is some positive $p$, and $\theta$ is a nonnegative function of $t$. Notice that other bounding functions might be possible.

4. Comparison theorem. In this section we will show that the function $\omega+U$ is an upper bound for $u$. The statement of this result is in the form of a comparison theorem. Several lemmas needed to prove Theorem 4.4 will be presented first. The first lemma is a maximum principle stated in the form most useful for the proof of the theorem. Lemma 4.2 demonstrates how to choose the parameters in $\omega$ so that this statement of the maximum principle is satisfied within the regions $\Omega_{0}(t):=$ $\{x \mid 0 \leq x \leq \Gamma(t)\}$ and $\Omega_{1}(t):=\{x \mid \Gamma(t)<x\}$. Finally, we see how the parameters in $\omega$ can be chosen to satisfy the maximum principle on $(x, t)=(\Gamma, t)$.

4.1. Maximum principle. This lemma is a modification of the Nagumo-Westphal Lemma [7] to include functions that are $C^{\infty}$ except on a set of measure zero where they may be only $C^{0}$. A condition on the spatial derivative replaces the condition involving a parabolic operator on this set. This lemma is a direct extension of the result by Nagumo and Westphal; thus, it is presented without proof.

LEMMA 4.1. Let $z(x, t)$ be a continuous function that is differentiable except on a finite number of curves, and let $u$ be continuous and differentiable everywhere. Suppose

$$
P[z] \geq P[u]
$$

in the regions where $z$ is differentiable, while $z$ satisfies

$$
(z)_{x}^{-}(X, t)=\lim _{x \uparrow X} \frac{\partial z(x, t)}{\partial x}>\lim _{x \downarrow X} \frac{\partial z(x, t)}{\partial x}=(z)_{x}^{+}(X, t)
$$




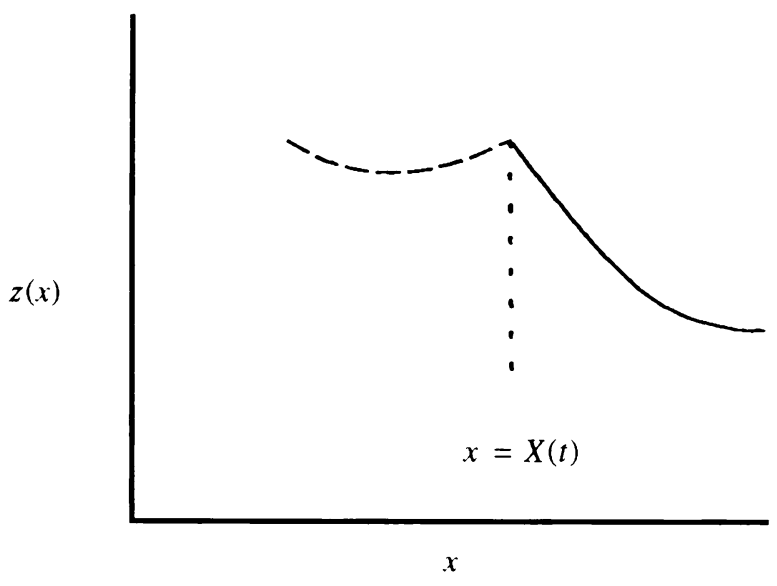

FiG. 1. Upper bounding function

for curves $(x, t)=(X(t), t)$ on which $z$ is continuous but not differentiable. When these conditions are satsified along with

$$
z \geq u \text { for }(x, t) \in \partial D
$$

then $z \geq u$ throughout $D$.

The type of bounding functions allowed by this lemma are illustrated in Fig. 1. The first derivative with respect to $x$ is larger on the left of $(x, t)=(X, t)$ than on the right.

4.2. Analysis of $\omega$ : Continuous regions. Suppose $F$ is convex; so

$$
f^{\prime}>\text { constant }
$$

for a positive constant independent of $\varepsilon$. We also assume that $f^{\prime}(v)$ is bounded above when its argument is bounded.

The shock speed $S(t)$ and the values of $f(u)$ are related by the mean value theorem

$$
S(t)=f(\widehat{U}),
$$

where $\widehat{U}(t)$ is the open interval $] U_{R}, U_{L}$ [. We will assume the slightly more restrictive case of $\widehat{U}$ in the closed interval $\left[U_{R}+\Delta, U_{L}-\Delta\right]$, where $\Delta$ is a positive constant. This relationship between $S$ and $f$ will be exploited in the proof of the following lemma.

LEMMA 4.2. There is an $\varepsilon_{0}$ small enough such that we may construct a specific form of $\omega$ to satisfy inequality (16) for region $\Omega_{1}$.

Proof. First some algebraic details are discussed. Let $\hat{x}=|x-\Gamma| / \nu$. The relationship between $\nu>0$ and $\varepsilon$ will be explained later. Differentiation of $\omega$ results in

$$
\begin{gathered}
\omega_{t}=a \nu+\left(\theta^{\prime}+\frac{\theta p K S}{\nu} \hat{x}^{p-1}\right) \exp \left(-K \hat{x}^{p}\right), \\
\omega_{x}=-\frac{\theta p K}{\nu} \hat{x}^{p-1} \exp \left(-K \hat{x}^{p}\right),
\end{gathered}
$$


and

$$
\omega_{x x}=\left(-\frac{\theta p(p-1) K}{\nu} \hat{x}^{p-2}+\frac{\theta p^{2} K^{2}}{\nu^{2}} \hat{x}^{2 p-1}\right) \exp \left(-K \hat{x}^{p}\right) .
$$

We use the mean value theorem to obtain $F(U+\omega)_{x}=f(U+\omega) \omega_{x}+(f(U)+$ $\left.\omega f^{\prime}\right) U_{x}$, where $f^{\prime}$ is evaluated between $U$ and $U+\omega$. The parabolic operator from Eq. (1) applied to the bounding function is

$$
P[U+\omega]=\omega_{t}+f \omega_{x}+f^{\prime} \omega U_{x}-\varepsilon U_{x x}-\varepsilon \omega_{x x}-R(U+\omega)
$$

or

$$
P[U+\omega]=\varepsilon \tau_{1}+\tau_{2}+\frac{\varepsilon}{\nu^{2}} \tau_{3}+\frac{1}{\nu} \tau_{4}
$$

where the terms have been grouped according to their magnitudes as

$$
\begin{aligned}
\tau_{1} & =\frac{\nu}{\varepsilon} a\left(1+t f^{\prime} U_{x}\right)-U_{x x}, \\
\tau_{2} & =\left[\theta^{\prime}+\theta f^{\prime} U_{x}\right] \exp \left(-K \hat{x}^{p}\right)-R, \\
\tau_{3} & =-\theta K^{2} p^{2} \hat{x}^{2 p-1} \exp \left(-K \hat{x}^{p}\right),
\end{aligned}
$$

and

$$
\tau_{4}=\theta K p\left[(S-f) \hat{x}^{p-1}+(p-1) \hat{x}^{p-2}\right] \exp \left(-K \hat{x}^{p}\right) .
$$

We will consider two cases based on the two forms of $\omega$ to verify that (16) is satisfied. First, choose $a$ large enough and $T$ small enough so that $\tau_{1}>0$.

Case I. When $x \in \Omega_{0}$ we let $\omega=\omega_{0}$, where

$$
\omega_{0}=a \varepsilon t
$$

Inequality (16) holds in this case since $\tau_{2}, \tau_{3}, \tau_{4}=0$.

Case II. When $x \in \Omega_{1}$ we let $\omega=\omega_{1}$, where

$$
\omega_{1}=a \varepsilon t+\mu \exp \left(-\kappa \hat{x}^{p}\right) .
$$

Here, $\delta$ and $\kappa$ are positive constants independent of $\varepsilon$. (Recall that $\mu$ is a positive function of $t$.) The term $\tau_{1}$ is positive from the choices of $a$ and $T$ above; thus, it is sufficient to consider only $\tau_{2}, \tau_{3}$, and $\tau_{4}$. We choose $\nu \gg \varepsilon$. For the sake of creating a constructive proof, we could use $\nu=\varepsilon|\ln (\varepsilon)|$; however, other choices for $\nu$ are possible. Then, we assume that $\varepsilon_{0}$ is small enough that with this choice of $\nu$, it is sufficient to show that $\tau_{4}$ is bounded below by a positive constant that is independent of $\varepsilon$. Here, we also restrict $p>1$ so that the second term of $\tau_{4}$ is positive.

First, we will establish a lower bound for $S(t)-f(U+\omega)$. These properties are based on the fact that $f(u)$ is the speed at which the characteristics of $u$ travel. The characteristics will be traveling faster than the shock for $x$ to the left of $\Gamma$, and slower than the shock for $x$ to the right of $\Gamma$. This means that when $\varepsilon$ is small enough, $S(t)-f(U+\omega)$ is positive for $\hat{x}>b_{0}(\kappa)$. We can assign a physical meaning to $b_{0}$ by interpreting it as being in the middle of the shock-layer. The relation between $\hat{x}$ and $S(t)-f(U+\omega)$ is depicted in Fig. 2. The precise shape of the curve cannot be known without more information about $f$; however, the location 


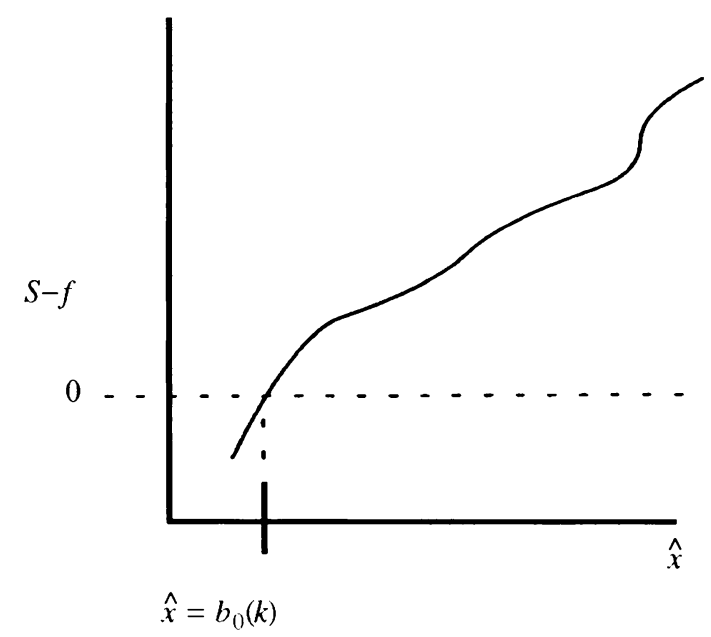

Fig. 2. Example form for $S(t)-f(U+\omega)$, with $t$ fixed

of $b_{0}(\kappa)$ moves closer to the origin as $\kappa$ increases. Multiplying $S-f$ by $\hat{x}$ reduces the variation near the origin, and we can choose $\kappa$ large enough such that

$$
1+(S(t)-f(U+\omega)) \hat{x}>\frac{1}{2}
$$

We now have specific forms for the bounding function in each of the domains. Next we must show that we can satisfy inequality (17) and $C^{0}$ continuity with $U+\omega$ with the scaling and $\kappa$ of the previous lemma.

4.3. Analysis of $\omega$ : Discontinuities. First we will establish the $C^{0}$ continuity by choosing the parameters for the bounding function. Condition (18) will also be satisfied by the choices in this lemma.

LemMa 4.3. Assume that the initial shock-layer profile is of exponential type. We may choose the parameters in $\omega_{1}$ to satisfy (18) and such that $U+\omega$ is $C^{0}$.

Proof. We use $\omega_{0}$ and $\omega_{1}$ as in Eqs. (20-21); thus $U+\omega$ is bounded. The result will follow providing (18) is satisfied for $(x, t) \in \partial D$. This relation holds except possibly for $(x, t) \in \pi_{0}$. In this region, we assume that the viscous profile of the shock-layer is of an exponential type that is bounded by the function $\omega$ presented in this proof. This is not a severe restriction, since a layer with a profile similar to that of $\tanh [-(x / \varepsilon)]$ (a solution to Burgers' equation) is suitable.

Theorem 4.4. Assume that $g$ and $g_{0}$ with their first and second derivatives are bounded independently of $\varepsilon$ except that $g_{0}$ has a jump at $(x, t)=\left(\Gamma_{0}, 0\right)$ and $g$ may have derivatives bounded depending on $\varepsilon$ for $(x, t) \in \pi_{0}$. Suppose $\omega$ is constructed from $\omega_{0}$ and $\omega_{1}$ as in Lemma 4.2. Also assume that $U_{x}$ is continuous across $(x, t)=(\Gamma, t)$. Then there is a positive $\varepsilon_{0}$ such that

$$
U+\omega \geq u
$$

for $(x, t) \in D$ when $\varepsilon_{0}>\varepsilon>0$.

Proof. We will have only one case to consider based on satisfying (17) on the curve $(x, t)=(\Gamma(t), t)$. Consider the modified function $\xi(x, t, \delta)=|x-\Gamma+\delta \nu| / \nu$. The 
use of this function allows us to move the location of the jump in the derivative of the bounding function in the positive $x$ direction. Observe that for positive $\delta$, the term $\omega_{x}$ is negative and has magnitude $\delta$, even when $\delta$ is asymptotically close to zero. Thus, we return to the original distance function by taking the $\operatorname{limit} \lim _{\delta \downarrow 0} \xi=\hat{x}$.

It may be possible to modify the assumption that $U_{x}$ is continuous across $(x, t)=$ $(\Gamma, t)$. This constraint was imposed so that $\delta$ could be taken asymptotically close to zero in the proof. A more careful analysis should allow this constraint to be eased.

COROLlaRY 4.5. Under the conditions of the theorem,

$$
|u-U|=O\left(\mu \exp \left[-\frac{\kappa|x-\Gamma(t)|}{\nu}\right]\right)+O(\varepsilon),
$$

when $\varepsilon_{0}>\varepsilon>0$ and $\nu(\varepsilon)$ is chosen as above.

This result follows directly by using symmetric arguments to obtain a lower bound, and choosing $p \geq 1$.

5. Implications. A direct result of this theorem is an upper bound on the size of the shock-layer. In this context, the shock-layer is defined as the region in which the solution to (1) differs from the solution to (2) by more than a specified amount. Namely, it is the region in which

$$
|u-U|>\sigma
$$

for some positive $\sigma$. As reflected in the following corollaries, there are different conclusions depending on whether one defines the location of the boundary of the shock-layer using $\sigma$ as a function of $\varepsilon$ or not. The following corollary treats the case when $\sigma$ is independent of $\varepsilon$ and follows directly from Corollary 4.5.

Corollary 5.1. Suppose the conditions of Theorem 4.5 obtain. If $\sigma$ is a constant independent of $\varepsilon$ then there is an $\varepsilon_{0}$ small enough so that $|u-U|<\sigma$ when

$$
|x-\Gamma(t)|=O(\nu)
$$

for $\varepsilon_{0}>\varepsilon>0$.

When we define the shock-layer as the region such that

$$
|u(x, t)-U(x, t)|>\varepsilon,
$$

then there is a positive $\varepsilon_{0}$ such that

$$
|x-\Gamma(t)|=O(\varepsilon \ln (\varepsilon))
$$

for $\varepsilon_{0}>\varepsilon>0$. Figure 3 depicts these ideas. 


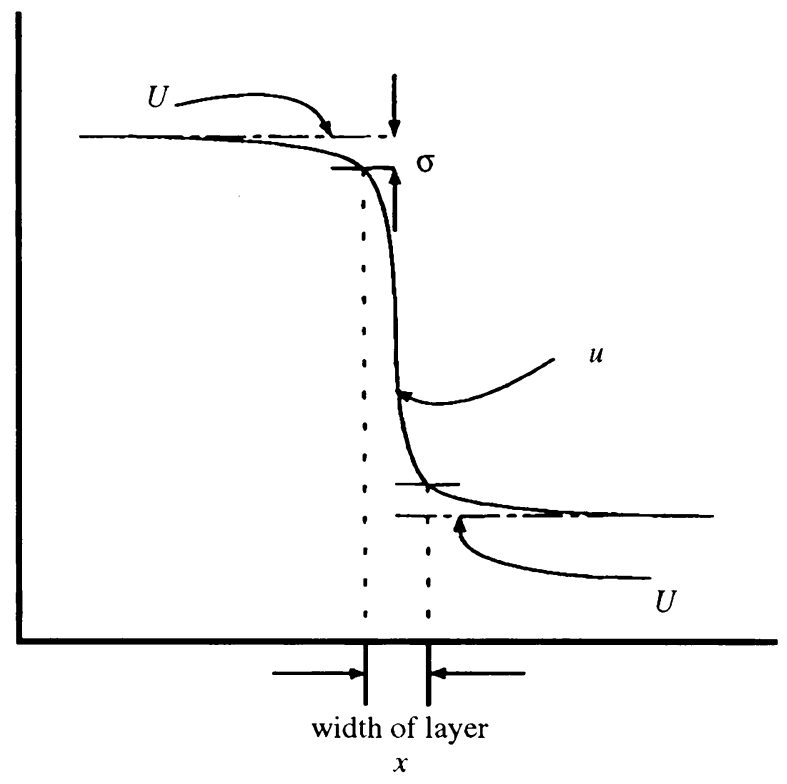

FIG. 3. Width of the shock-layer at fixed $t$

6. Concluding remarks. We have used brute-force asymptotic analysis to derive the candidate form for the bounding function (15). Asymptotic analysis could be used to provide more insight into the behavior of the functions; however, the results here are sharp. Suppose $u=\tanh [(x-\Gamma) / \varepsilon]$. Then the size of the internal-layer subdomain is still $O(\varepsilon \ln \varepsilon)$. Thus, we could have worked harder, but would not have sharpened the bound on the size of the layer.

\section{REFERENCES}

[1] J. R. Cannon, The One-Dimensional Heat Equation, vol. 23, Addison-Wesley Publishing Company, Reading, Massachusetts, 1984

[2] F. A. Howes, Perturbed boundary value problems whose reduced solutions are nonsmooth, Indiana Univ. Math. J. 30, 267-280 (1981)

[3] F. A. Howes, Multi-dimensional reaction-convection-diffusion equations, in Ordinary and Partial Differential Equations, Proceedings of the Eighth Conference, Dundee, Scotland, B. D. Sleeman and R. J. Jarvis, eds., vol. 1151, Springer-Verlag, New York, 1984, pp. 217-223

[4] F. A. Howes, Multi-dimensional initial-boundary value problems with strong nonlinearities, Arch. Rat. Mech. Anal. 91, 153-168 (1986)

[5] F. A. Howes, Nonlinear initial-boundary value problems in Rn, Lectures in Applied Mathematics 23, 259-273 (1986)

[6] P. D. Lax, Hyperbolic Systems of Conservation Laws and the Mathematical Theory of Shock Waves, SIAM, Philadelphia, 1973

[7] W. Walter, Differential and Integral Inequalities, Springer-Verlag, New York, 1970 